\title{
Open charm reconstruction at mid-rapidity in the ALICE Experiment at LHC
}

\section{Sadhana Dash (for the ALICE Collaboraton)*}

Universita and INFN di Torino, ITALY

E-mail: dash@to.infn.it

\begin{abstract}
Open charmed mesons produced in ultra-relativistic nuclear reactions are among the most direct probes to investigate the medium produced in these collisions. It is important to measure the production of as many charmed hadrons as possible, such as $D^{0}, D^{+}, D_{s}, D^{*}$ and $\Lambda_{c}$, because the measurement of their relative yield can provide information on the hadronization mechanism. It will also reduce the systematic error on the total charm cross-section. The ALICE experiment at the LHC is designed to perform such measurements at mid-rapidity by means of its barrel tracking detectors. The study on exclusive reconstruction of $D^{0}, D^{+}$and $D^{*+}$ mesons through their hadronic decay channels will be presented for $p p$ collisions at $7 \mathrm{TeV}$. The first results on D-meson spectra and the $\mathrm{D}$ meson ratios will also be shown.
\end{abstract}

XLIX International Winter Meeting on Nuclear Physics

24-28 January 2011

BORMIO, Italy

${ }^{*}$ Speaker. 


\section{Introduction}

The main motivation for studying heavy-ion collisions at high energy is the study of Quantum Chromodynamics (QCD) in extreme conditions of high temperature and high energy density [1]. These collisions create nuclear matter of high energy density over an extended volume, allowing QCD predictions to be tested in the laboratory. At high temperature and density, QCD predicts a phase transition from nuclear matter to a state of deconfined quarks and gluons known as the quark gluon plasma (QGP). Heavy, long lived quarks, i.e. charm and bottom, are frequently studied both as tests of QCD and as probes for other physics aspects within and beyond standard model. They are sensitive probes of the medium produced in nucleus-nucleus collisions. They are predominantly produced by initial hard parton scattering processes and thus, the initially produced heavy quarks experience the full collision history. Both the initial and final state effects of the medium are expected to enter in the production and propagation of heavy quarks. Perturbative QCD calculations at fixed order plus next to leading log (FONLL) describe well the cross section of charm and bottom particles. The world data (single electrons from STAR/PHENIX experiments at RHIC and D mesons from CDF) are described by the upper limit of the FONLL calculations $[2,3,4,5]$. The measurement of open charm in $p p$ collisions at LHC energies is important to test the predictions of pQCD calculations in the new energy domain. Furthermore, the yields of open charm will act as reference for the study of in-medium effects in the system produced in $P b-P b$ collisions.

Beyond the total charm cross-section, open charmed mesons can be used to probe the medium produced in relativistic heavy-ion collisions. The medium-induced suppression of high $p_{t}$ particles in nucleus-nucleus collisions can be characterized by the nuclear modification factor, $R_{A A}$. For example, the suppression of $R_{A A}$ from semi-leptonic D and B-meson decay in central $A u-A u$ collisions was expected to be less severe for charm quarks than for lighter flavors because of the dead cone effect (where gluon radiation is suppressed because of destructive interference for angles less than $m / E, \mathrm{~m}$ and $\mathrm{E}$ are the mass and energy of the quark [6]). Consequently a decrease of high $p_{t}$ suppression is expected from light to charm to bottom quarks. In contrast, a strong suppression of heavy flavour decay electrons was discovered. Recent experimental results from RHIC (STAR and PHENIX) experiment observed large yield suppression of heavy flavor decay electrons in central $A u-A u$ collisions, consistent with substantial energy loss of heavy quarks in dense matter $[4,7,5]$. The suppression is larger than that expected from radiative energy loss calculations, suggesting that other processes contribute significantly to heavy quark energy loss. This unique sensitivity to the energy loss mechanisms make the measurement of heavy quark suppression an essential component of the study of dense matter.

This paper is organized as follows. The section 2 contains a brief description of the ALICE experimental setup. The section 3 describes the analysis strategy followed by some preliminary results in section 4 . The results are summarized in section 5 .

\section{The ALICE Experimental setup}

A Large Ion Collider Experiment, ALICE is a general-purpose heavy ion detector at the CERN LHC designed to address the physics of strongly interacting matter and the quark gluon plasma at extreme values of energy density and temperature in nucleus nucleus collisions. The ALICE 


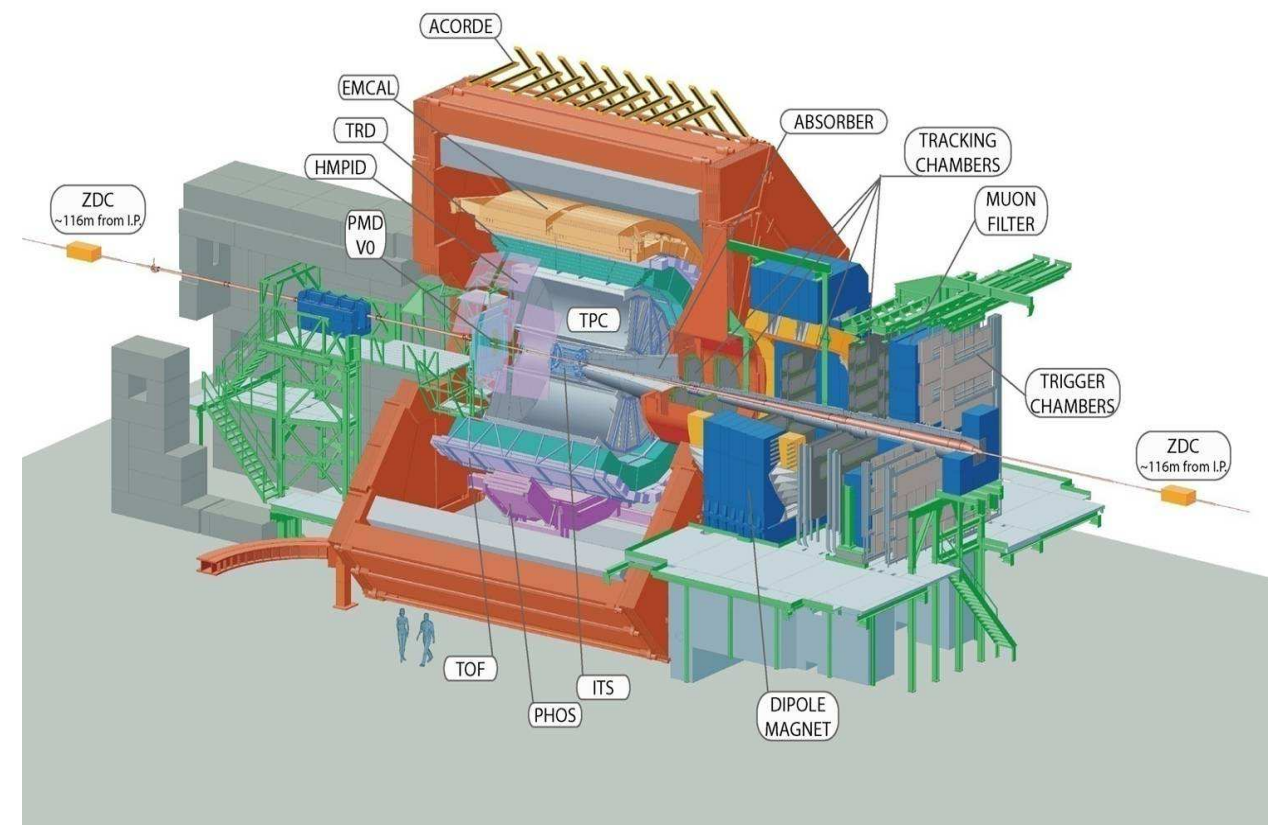

Figure 1: The ALICE experimental setup. See text for details.

setup consists of a central barrel covering the full azimuthal angle in $|\eta|<0.9$ and several forward systems. The central barrel system is embedded in a magnetic field, B of $0.5 \mathrm{~T}$. The central detectors are, in the order from the interaction vertex to the outside, the Inner Tracking System (ITS) with six layers of silicon detectors, the Time Projection Chamber (TPC), which is the main tracking detector, the Transition Radiation Detector (TRD) for electron identification and the Time Of Flight detector (TOF), which extends the momentum range of particle identification (PID) up to $4 \mathrm{GeV} / \mathrm{c}$. The Time-Projection Chamber (TPC) is the main tracking detector of the central barrel and is optimized to provide, together with the other central barrel detectors, charged-particle momentum measurements with good two-track separation, particle identification, and vertex determination.

Two other detectors with reduced acceptance complement the central barrel: the High-Momentum Particle Identification Detector (HMPID) consisting of an array of ring-imaging Cherenkov counters and the Photon Spectrometer (PHOS), which is an electromagnetic calorimeter. The last addition to the ALICE design is the Electromagnetic Calorimeter (EMCal), dedicated to the physics of jets and high- $p_{t}$ photons. The EMCal also measures the light mesons, such as $\pi^{0}$ and $\eta$, decaying into photon. More technical detains on the design of the ALICE detector can be found in [8]. A schematic of the ALICE experimental setup is shown in Figure 1. The main detectors contributing to the study of charm hadrons are the ITS, TPC and TOF. The ITS with its impact parameter resolution of around $75 \mu \mathrm{m}$ at $1 \mathrm{GeV} / \mathrm{c}$ provides information about the secondary vertex, which is crucial for physics studies dependent on displaced vertex topologies. The resolution of impact parameter as a function of $p_{t}$ is shown in Figure 2.

The TPC and TOF signals are used for particle identifications as a function of transverse 


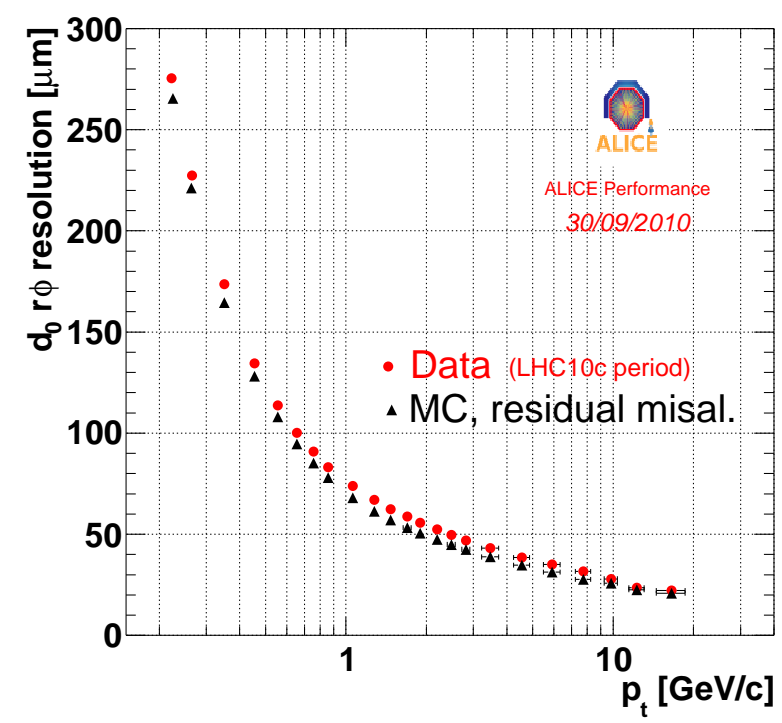

Figure 2: Transverse impact parameter resolution as a function of $p_{t}$.

momentum. The particle identification in the TPC is based on the specific ionization energy loss $(d E / d x)$ of each particle in the fill gas of the TPC. This is shown in Figure 3 (Upper panel) as a function of momentum. The particle identification at higher $p_{t}$ is assured by adding the information from the TOF detector where particles are identified by measuring simultaneously their momentum and velocity. The velocity of the particle is obtained from the reconstructed flight path and the time of flight. This is shown as a function of the momentum in Figure 3 (Lower panel). The bands corresponding to the charged pions, kaons and protons are clearly visible.

\subsection{Trigger Selection}

The main trigger selection is the minimum-bias, $\mathrm{MB}$, which is defined by the inclusion of at least one signal in either of the two scintillator hodoscopes, the V0 detectors, positioned in the forward and backward regions of the ALICE experiment or in the silicon pixel detector, SPD. This trigger selects events with at least one charged particle in the region of $|\eta|<8.0$ and is sensitive to about $95 \%$ of the $p p$ inelastic collisions. Most of the data is shown for $1.01 \times 10^{8}$ minimum-bias events.

\section{Reconstruction of Charmed mesons}

Open charm hadrons are not directly observable as they decay far too quickly (with $c \tau$ values of few hundred microns) to survive into the detectors. In order to study open charm mesons, they must be reconstructed via their hadronic decay channels. The selection strategy is based on the displaced vertex topology of the D meson decay. A single track is required to have at least 70 spatial points in TPC and should have at least 3 clusters in the ITS. All tracks that are identified as pions and kaons are combined into triplets or pairs with the correct combination of charge signs. 

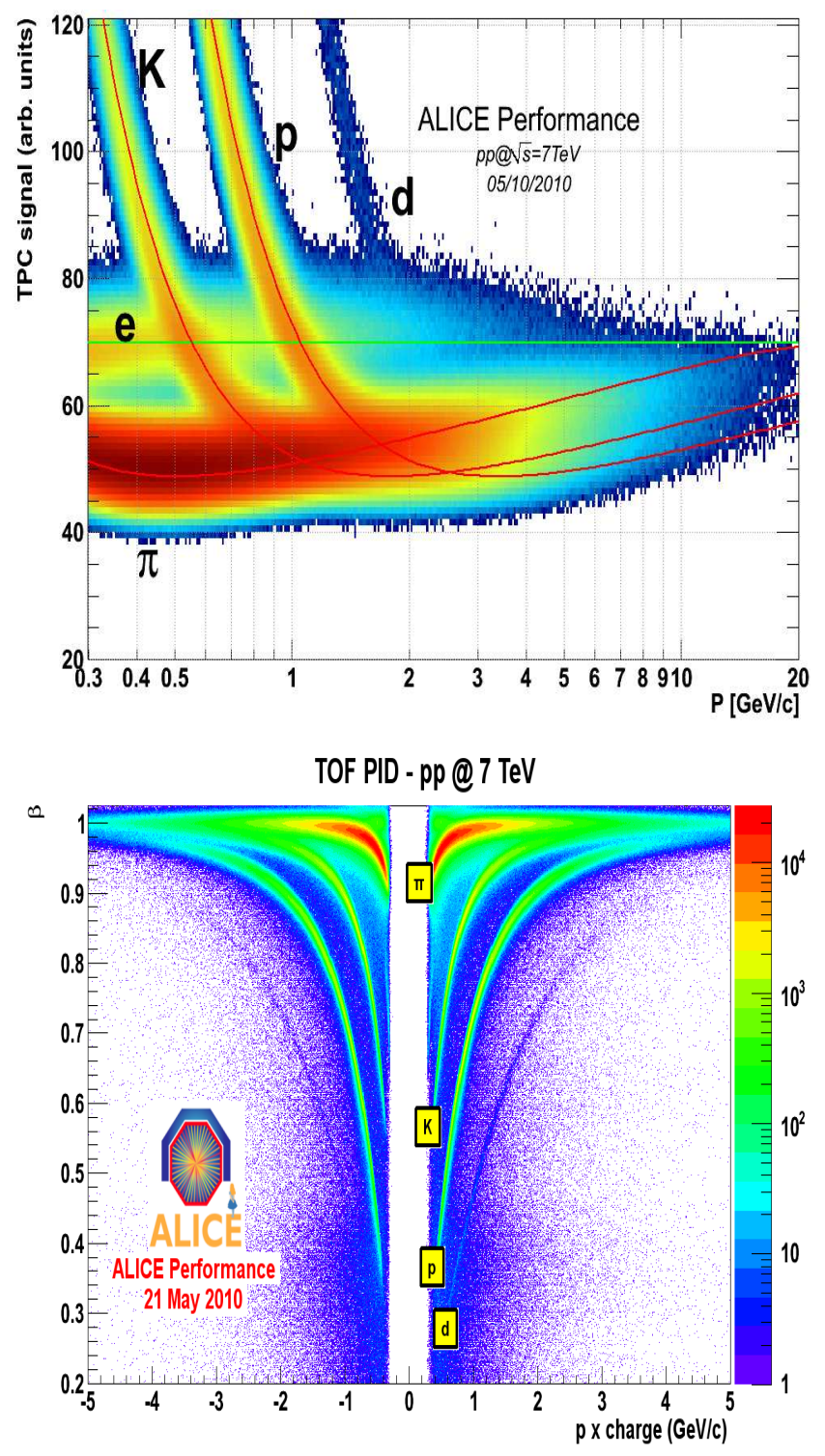

Figure 3: (Upper panel) The measured energy-deposit of charged particles versus the track momentum. The lines correspond to the ALEPH parameterization of the Bethe-Bloch curve. (Lower panel) The particle velocity as a function of the momentum measured by the TOF detector.

Then an invariant mass spectrum of all the candidate combinations is calculated to extract the raw yield.

One has to find some properties of the candidate pairs/triplets that allow distinguishing them from the combinatorial background and applying cuts on these variables. The task is to find optimum tuning for the cut values. The distance between primary vertex of the collision and decay vertex of the meson is a good example of such a variable because the ITS detector of ALICE measures this distance with a good accuracy separating particles coming directly from primary vertex from those produced in secondary decays of short-lived mesons. 

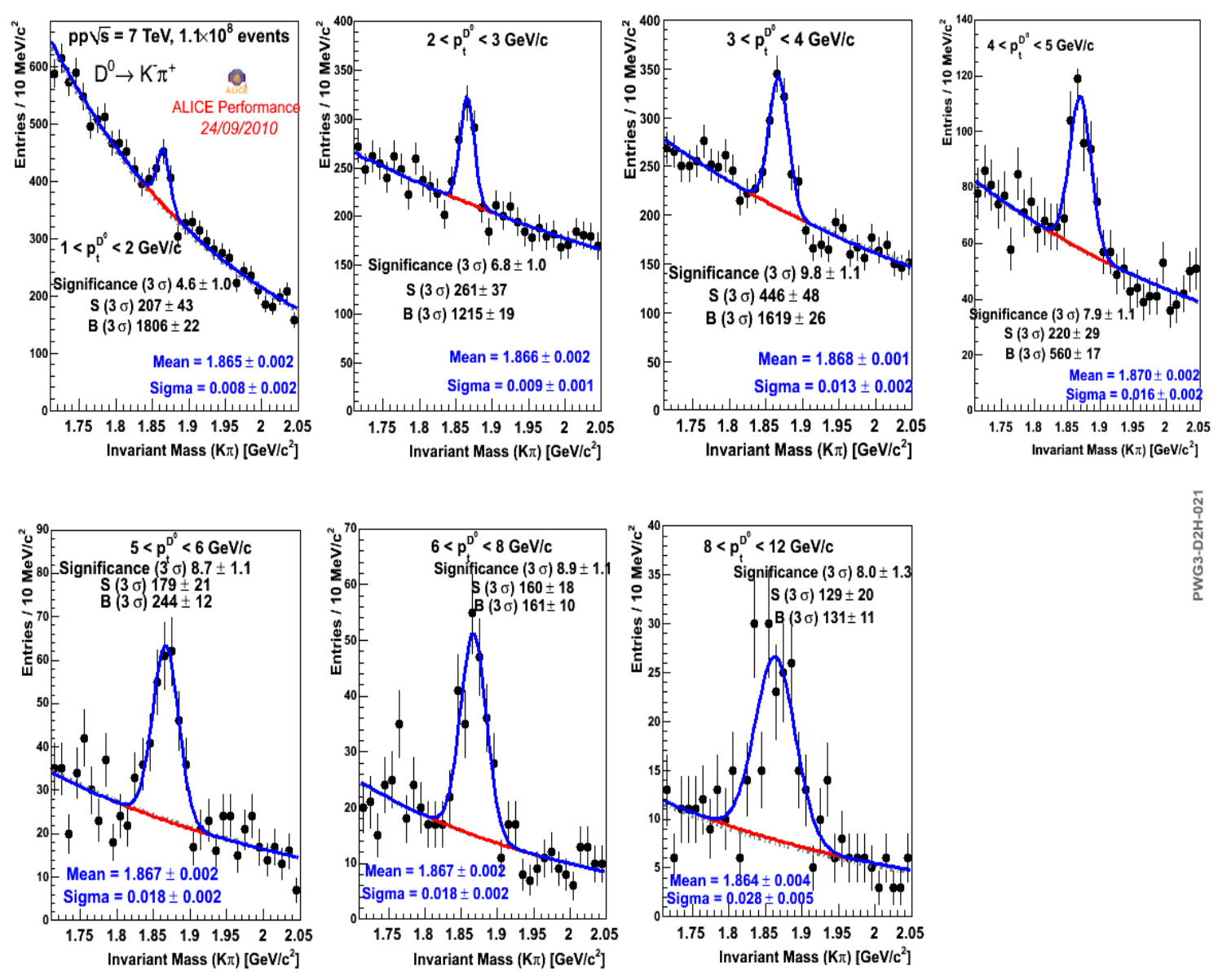

Figure 4: $K \pi$ invariant mass spectra for $D^{0}$ candidates in different $p_{t}$ bins.

\subsection{Selection Strategy}

The first step of the selection is applied at the level of single reconstructed tracks before combining them into pairs/triplets. At this stage, the selection is based on transverse momentum, $p_{t}$ and the impact parameter, $d_{0}$. The tracks that have passed the previous selections are combined into pairs with opposite charge sign. The vertex of these pairs of tracks is calculated using the standard ALICE vertexing algorithm. For the $D^{0}$ reconstruction in the channel $D^{0} \rightarrow K^{+} \pi^{-}(\mathrm{BR}=$ $3.80 \pm 0.05 \%)$, the relevant cut variables are the product of the impact parameters of the kaon and the pion track, cosine of the pointing angle, the difference between the mass of $D^{0}$ meson and the invariant mass of $K \pi$ pair and the distance between the found vertex and the primary vertex. The invariant mass distributions obtained in various $p_{T}$ bins for the $D^{0}$ meson is shown in Figure 4.

For the $D^{*+}\left(D^{*+} \rightarrow D^{0}+\pi^{+}, \mathrm{BR}=67.7 \pm 0.05 \%\right)$ meson, the invariant mass calculation is performed with a $D^{0}$ candidate and a soft pion. The background is estimated using the $D^{0}$ candidates with invariant mass falling in the side-bands $\left(4 \sigma-8 \sigma\right.$ range) of the $D^{0}$ mass region. The corresponding invariant mass distributions of $\mathrm{M}(K \pi \pi)-\mathrm{M}(K \pi)$ can be seen in Figure 5 . 

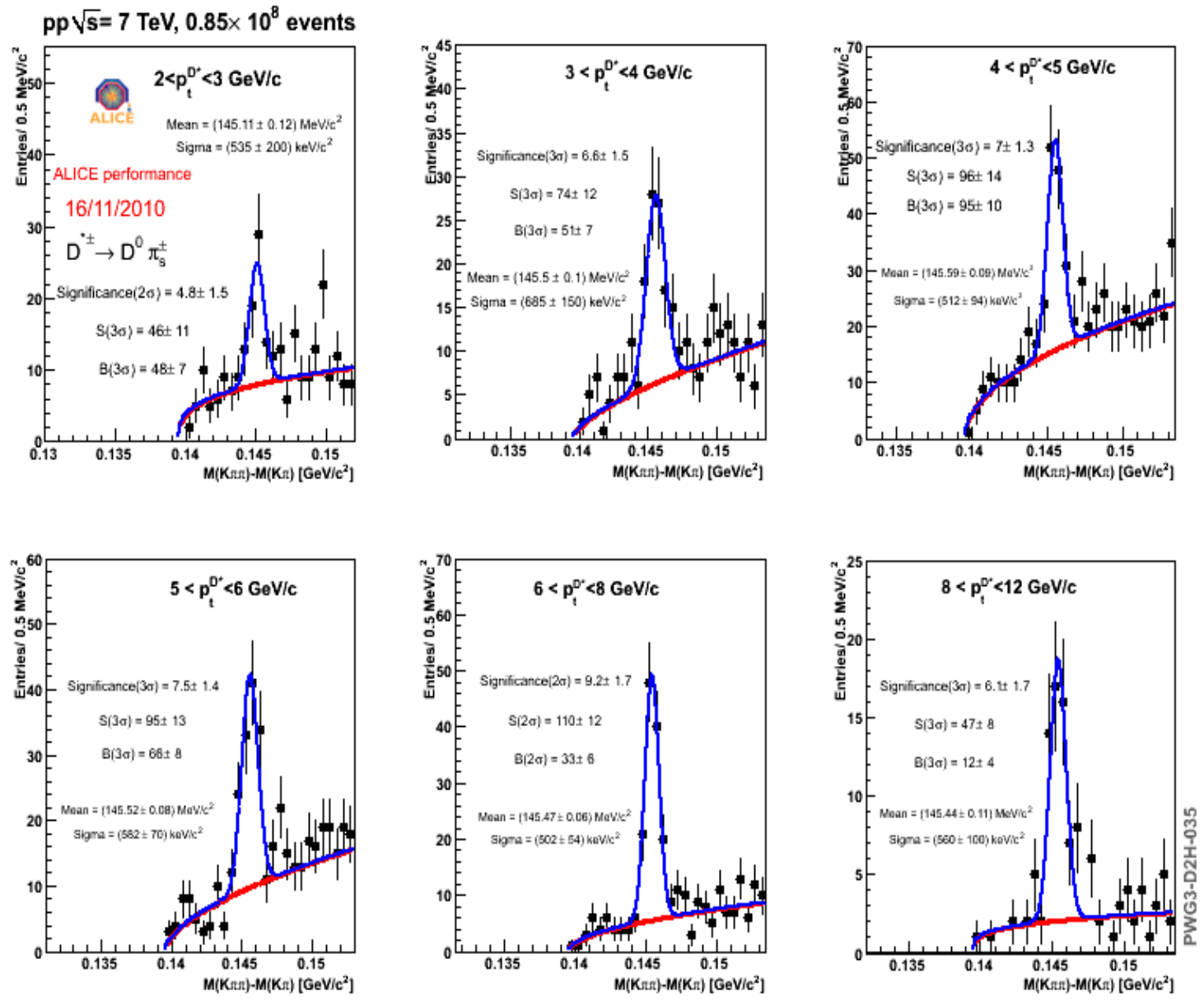

Figure 5: $K \pi \pi-K \pi$ invariant mass difference spectrum for $D^{*}$ candidates for various $p_{t}$ bins.

The reconstruction of $D^{+}$mesons in the channel $D^{+} \rightarrow \pi^{+} K^{-} \pi^{+}(\mathrm{BR}=9.20 \pm 0.05 \%)$ requires further cuts on the candidate triplets in order to enhance the signal to background ratio. The cuts are namely the distance among the tracks and found vertex, $\cos \left(\theta_{\text {point }}\right)$ where $\theta_{\text {point }}$ is the pointing angle (the angle between the direction of the reconstructed $D^{+}$momentum in the bending plane and the line connecting the primary and secondary vertices), the sum of the squares of the three tracks impact parameters with respect to the primary vertex (this cut is based on the fact the signal tracks are coming from the displaced secondary vertex), distance between the primary and secondary vertices and the quality of the found secondary vertex (based on the dispersion of three tracks around the vertex). After applying these track pair and triplet selections, an invariant mass of the $\pi K \pi$ triplet is calculated for raw yield extraction. This is shown in Figure 6.

The invariant mass distribution of other promising open charm signals seen in rare decay channels of $D_{s}^{+} \rightarrow K^{+} K^{-} \pi^{+}, \Lambda_{c}^{+} \rightarrow \pi^{-} p K^{+}$and $D^{0} \rightarrow \pi^{+} \pi^{-} \pi^{+} K^{-}$is depicted in Figure 7.

\subsection{Efficiency Corrections}

The raw signal yield extracted from the invariant mass distribution is a small fraction of the 

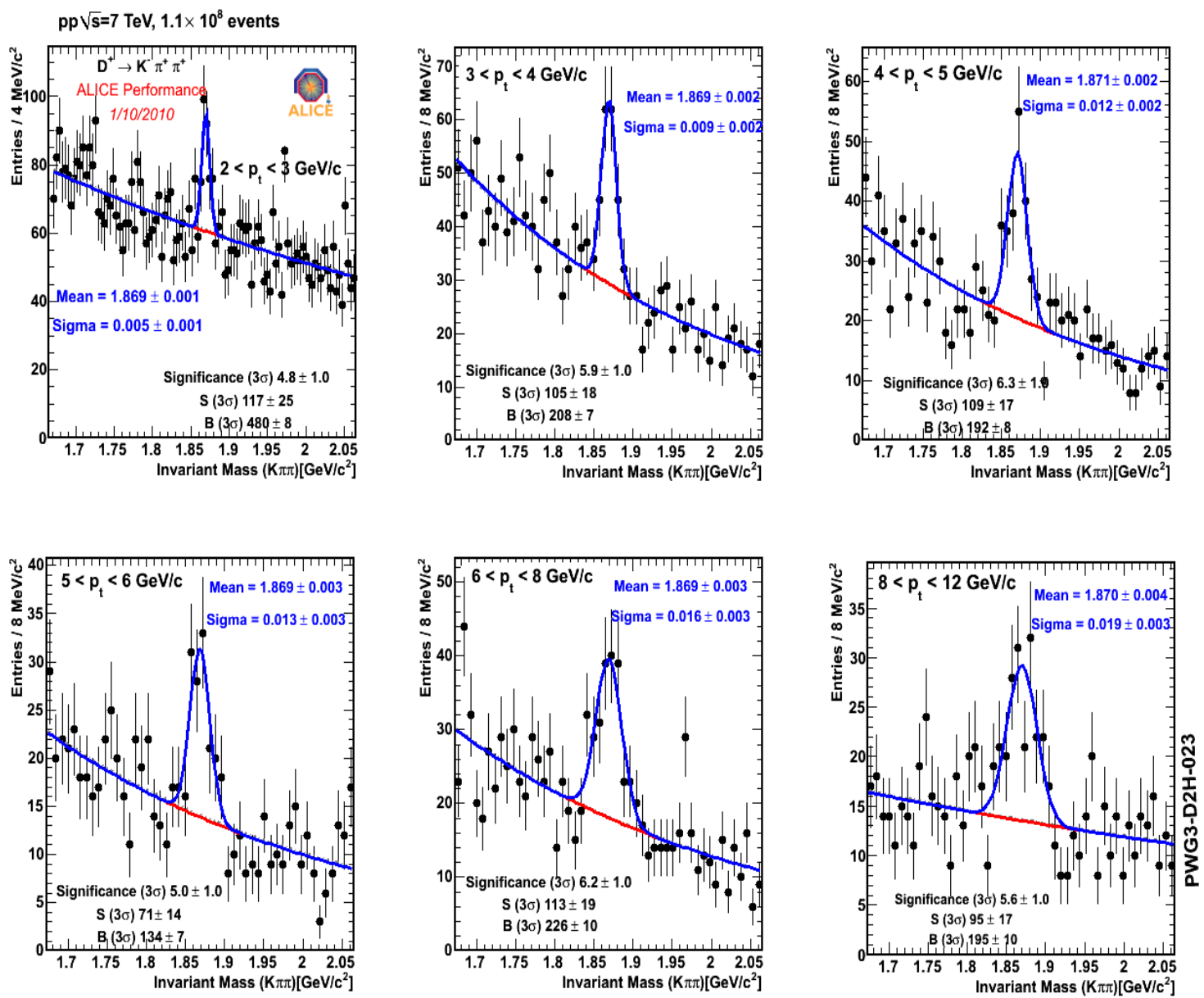

Figure 6: $K \pi \pi$ invariant mass spectra for $D^{+}$candidates in different $p_{t}$ bins.

total number of $\mathrm{D}$ mesons produced due to detector acceptance, vertexing and tracking inefficiencies and various selection cuts on tracks. The D meson invariant yields as a function of $p_{t}$ are then corrected for detector acceptance and reconstruction efficiency. The efficiency factors were extracted from Monte Carlo simulations with detailed description of detector response and experimental conditions for $p p$ collisions. The variation of the efficiency with $p_{t}$ for $D^{0}, D^{+}$and $D^{*+}$ mesons is depicted in Figure 8.

At LHC energies, a significant fraction of D meson comes from the decay of B mesons. This contribution must be estimated and subtracted from the raw yield in order to obtain the production cross section for prompt charm. In the present analysis, the correction factor for bottom quark feed down is estimated using the bottom production cross-section predictions of FONLL calculations with detector simulation. This estimation will be checked with the data driven method developed by the CDF Collaboration [3] with full available statistics. The systematic uncertainties on the D meson cross-sections were estimated as follows: (1) An uncertainty on raw yield extraction, which was evaluated by repeating the fit, in each $p_{t}$ interval, in a different mass range and with a different function describing the background. (2)The uncertainties due to efficiency correction 

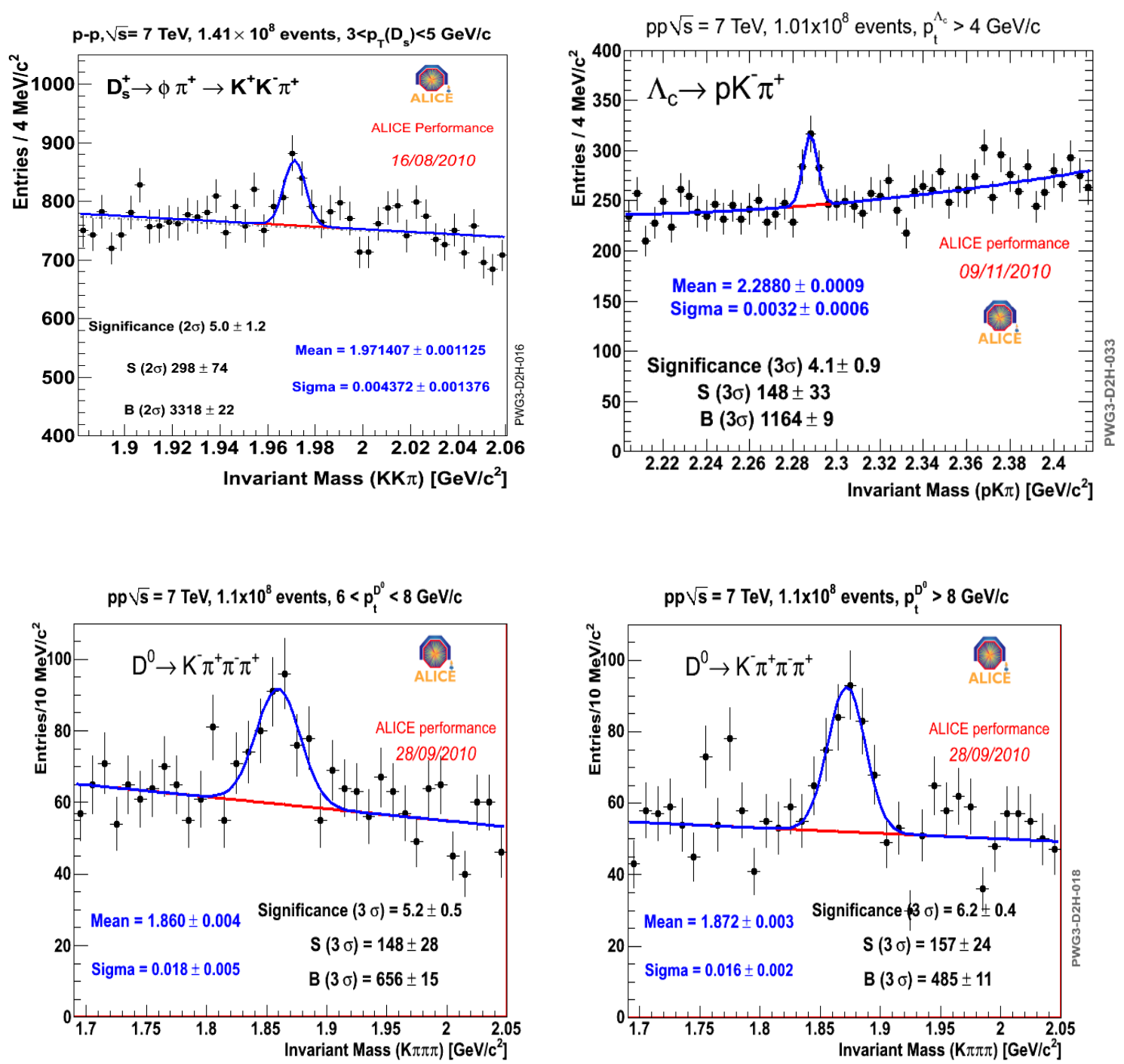

Figure 7: The invariant mass spectra showing $D_{s}, \Lambda_{c}$, and $D^{0}\left(D^{0} \rightarrow \pi^{+} \pi^{-} \pi^{+} K^{-}\right)$signals.

factors and the beauty feed down factors. The total systematic uncertainties amounted to 20-25\% . No significant $p_{t}$ dependence of the systematic uncertainties is observed for $p_{t}>3 \mathrm{GeV} / \mathrm{c}$. The systematic uncertainties arising from the different sources for the three mesons are shown in Figure 9.

\section{Results}

After the raw yield extraction and corrections for various factors mentioned in the previous section, the D meson cross-section is calculated using the following formula:

$$
\left.\frac{d \sigma}{\mathrm{dp}_{\mathrm{t}}}\right|_{|\mathrm{y}|<0.5}=\frac{1}{2} \cdot \frac{1}{\Delta \mathrm{y}\left(\mathrm{p}_{\mathrm{t}}\right)} \cdot \frac{1}{B R} \cdot \frac{1}{\varepsilon_{c}} \cdot f_{c}\left(p_{t}\right) \cdot \frac{\left.\mathrm{N}_{\mathrm{Raw}}^{\mathrm{D}}\left(\mathrm{p}_{\mathrm{t}}\right)\right|_{|\mathrm{y}|<\Delta \mathrm{y}\left(\mathrm{p}_{\mathrm{t}}\right)}}{\mathrm{N}_{\text {MinBias }}^{\mathrm{tot}}} \cdot \sigma_{\text {MinBias }}^{\text {tot }}
$$



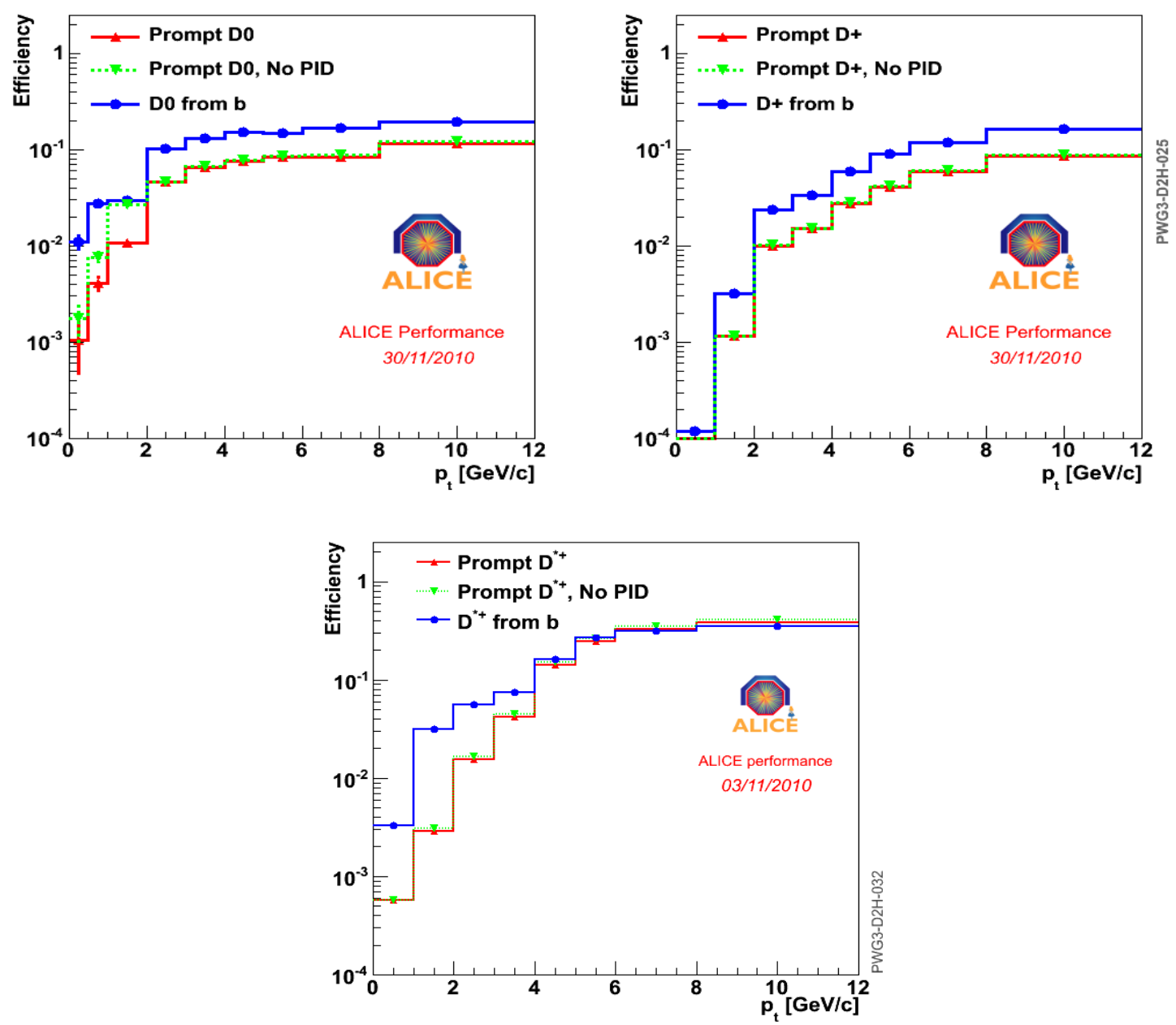

Figure 8: (Upper panel) $D^{0}$ and $D^{+}$reconstruction and selection efficiencies versus $p_{t}$. (Lower panel) $D^{*+}$ reconstruction and selection efficiencies versus $p_{t}$.

where $\mathrm{N}_{\text {Raw }}^{\mathrm{D}}\left(\mathrm{p}_{\mathrm{t}}\right)$ is the raw extracted yield, $\varepsilon_{c}$, the efficiency correction factor, $f_{c}$, the correction factor for the beauty feed-down and $\sigma_{\text {MinBias }}^{\text {tot }}$ is the total $p p$ inelastic cross-section for minimum bias events. The BR corresponds to the branching ratio of the decay channel studied while $\Delta y\left(p_{t}\right)$ is the rapidity acceptance. $\mathrm{N}_{\text {MinBias }}^{\text {tot }}$ is the total number of minimum-bias events. Figure 10 shows the preliminary $p_{t}$ differential cross section for $\mathrm{D}$ mesons compared with the theoretical predictions from FONLL and GM-VFNS. The data are well described by both calculations within theoretical uncertainties. Figure 11 illustrates a comparison of the $\mathrm{D}$ meson ratios, $D^{0} / D^{+}$and $D^{0} / D^{*+}$ for $p_{t}>3 \mathrm{GeV} / \mathrm{c}$, which are found to be in good agreement with previous measurements at low energies $[11,3]$.

\section{Summary and Outlook}

The first open charm production measurements performed by the ALICE detector in $p p$ colli- 

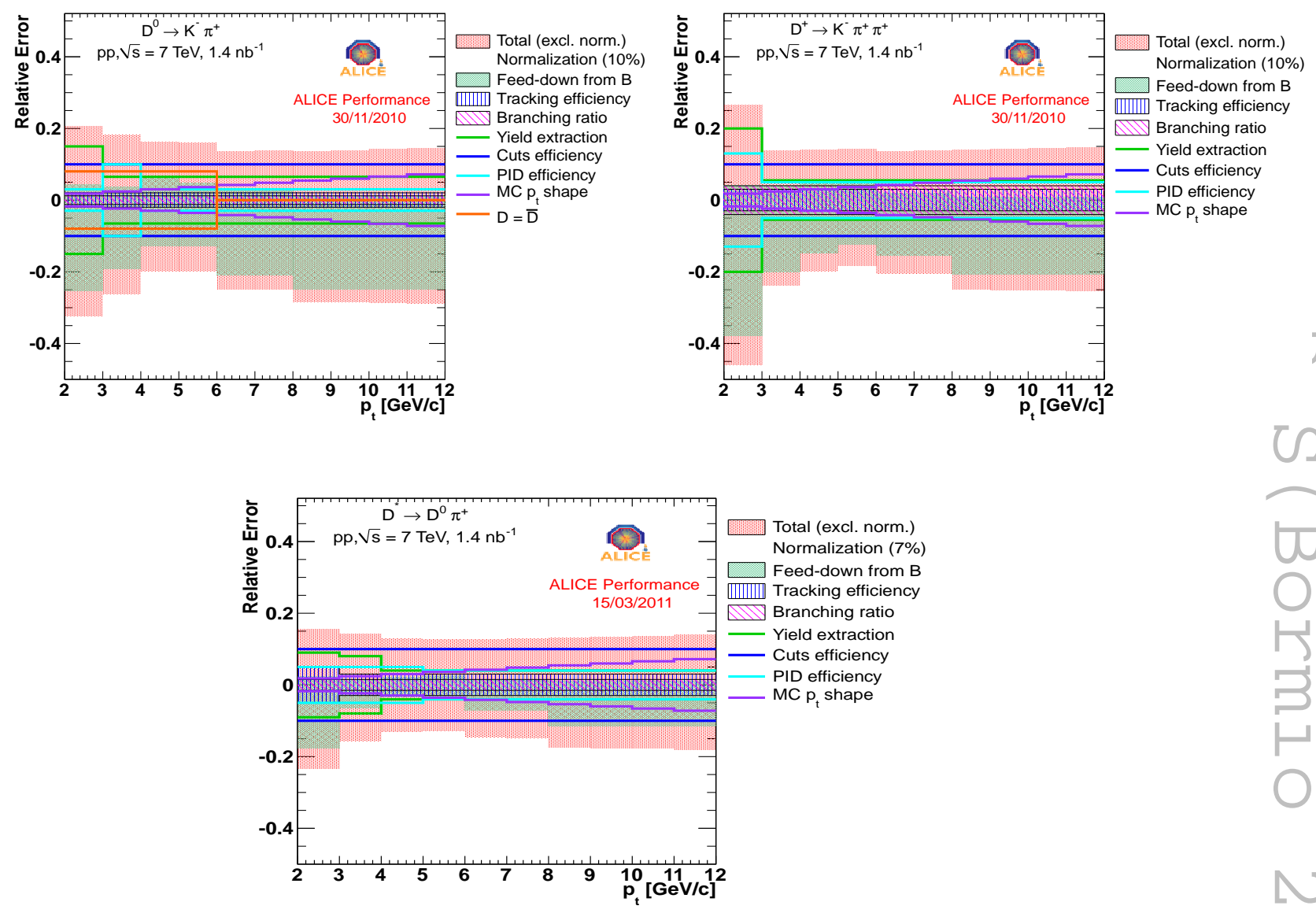

Figure 9: $D^{0}, D^{+}$and $D^{*+}$ mesons systematic errors corresponding to the preliminary cross-section results shown in Figure 10.

sions at $7 \mathrm{TeV}$ at $\mathrm{LHC}$ is presented at central rapidity. The excellent tracking, vertexing and particle identification capabilities of the ALICE detector allow the high precision measurement of the Dmeson cross section through its hadronic decay channels. The preliminary results on the production cross-section of the $D^{0}, D^{+}$and $D^{*+}$ mesons are well described by pQCD calculations within uncertainties. These measurements will provide useful reference for the upcoming measurements in heavy ion collisions at the LHC.

\section{References}

[1] J. Adams et al., STAR Collaboration, Nucl. Phys. A 757 (2005) 102.

[2] M. Cacciari, P. Nason, and R. Vogt, Phys. Rev. Lett. 95 (2005) 122001.

[3] D. Acosta et al., CDF Collaboration, Phys. Rev. Lett. 91 (2003) 241804.

[4] J. Adams, Phys. Rev. Lett. 91 (2003) 172302.

[5] A. Adare, Phys. Rev. Lett. 98 (2007) 172301. 

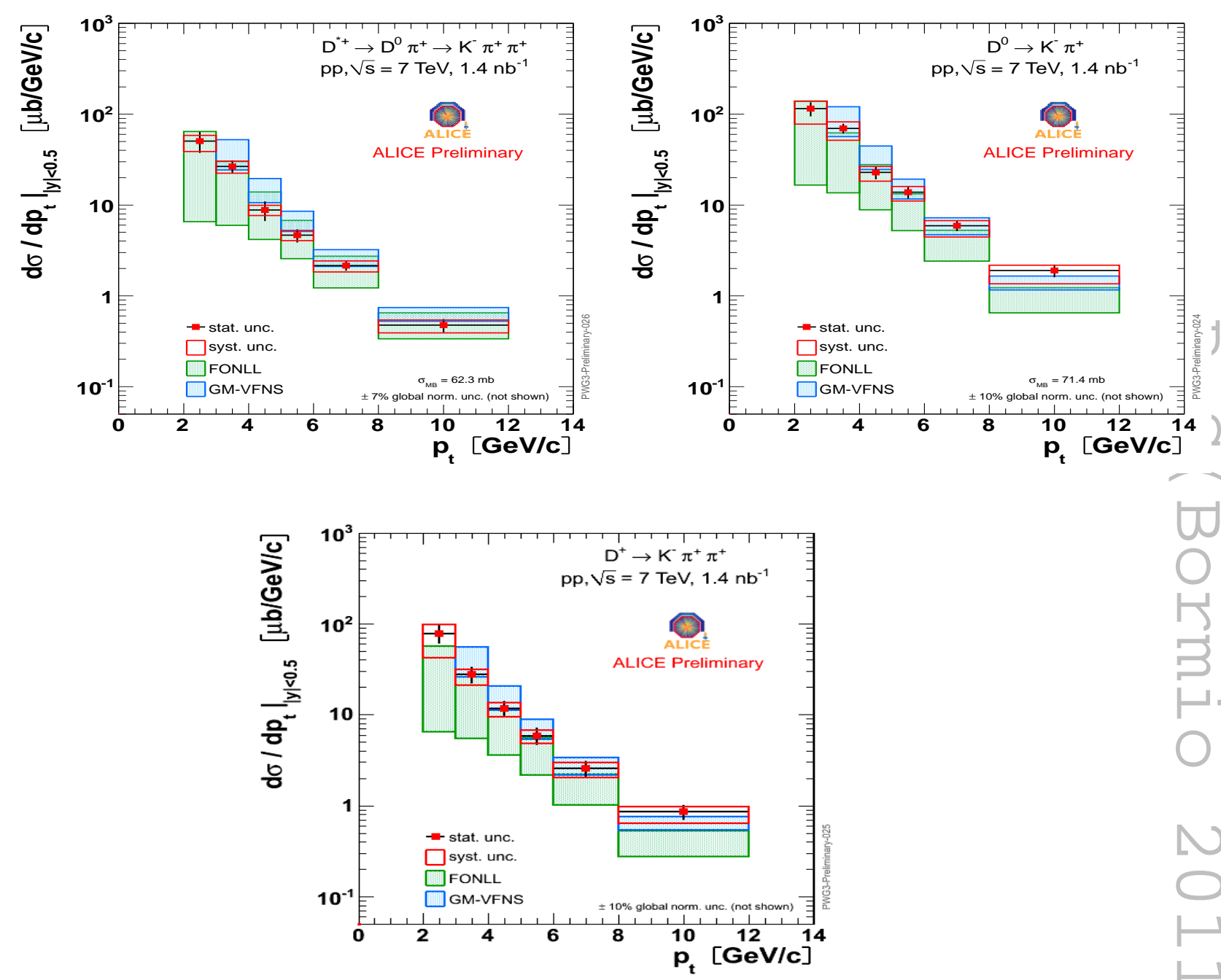

Figure 10: Preliminary $p_{T}$ differential cross section for $D^{*+}, D^{0}$ and $D^{+}$mesons, compared to predictions from FONLL([9]) and GM-VFNS([10]) calculations.

[6] Y.L. Dokshitzer and D.E. Kharzeev, Phys. Lett. B 519 (2001) 199.

[7] J. Schaffner-Bielich, Phys. Rev. Lett. 105 (2010) 202301.

[8] K. Aamodt et al., ALICE Collaboration, JINST 0803 (2008) S08002.

[9] M. Cacciari et al., Private Communication.

[10] B.A. Kniehl et al., Private Communication.

[11] S. Chekanov et al., ZEUS Collaboration, JHEP07 (2007) 074. 

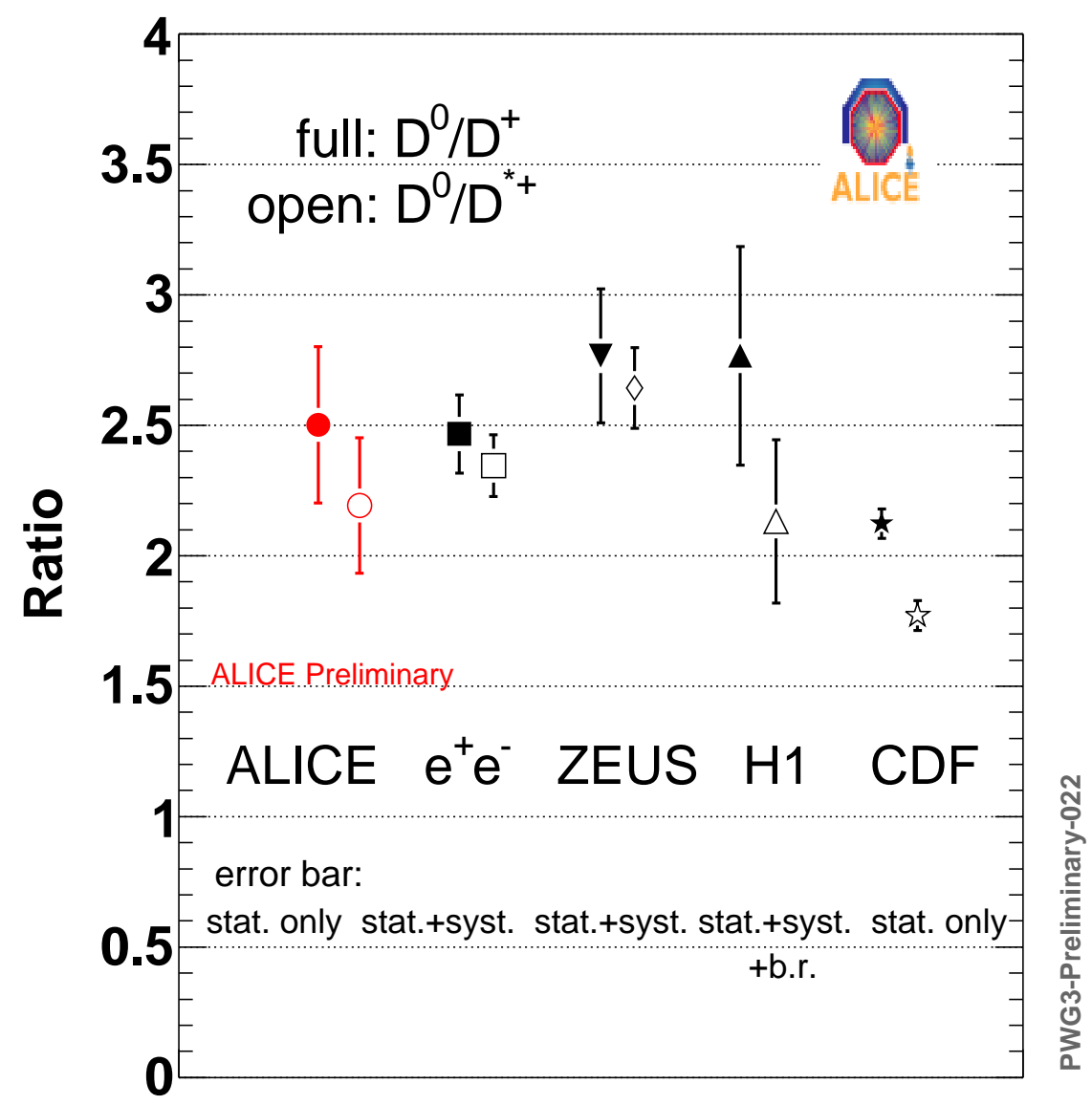

Figure 11: $D^{0} / D^{+}$and $D^{0} / D^{*+}$ ratios (in the range $3<p_{t}<12 \mathrm{GeV} / \mathrm{c}$ ) compared to previous experiments at lower collision energies. 